\title{
Progetto complesso per una storia complessa. L'alto Adriatico nel Novecento
}

\author{
di Luciana Rocchi
}

Ott 28, $2020|\underline{\text { In evidenza, }} \underline{\text { Pensare la didattica }}| \underline{0} \mid$

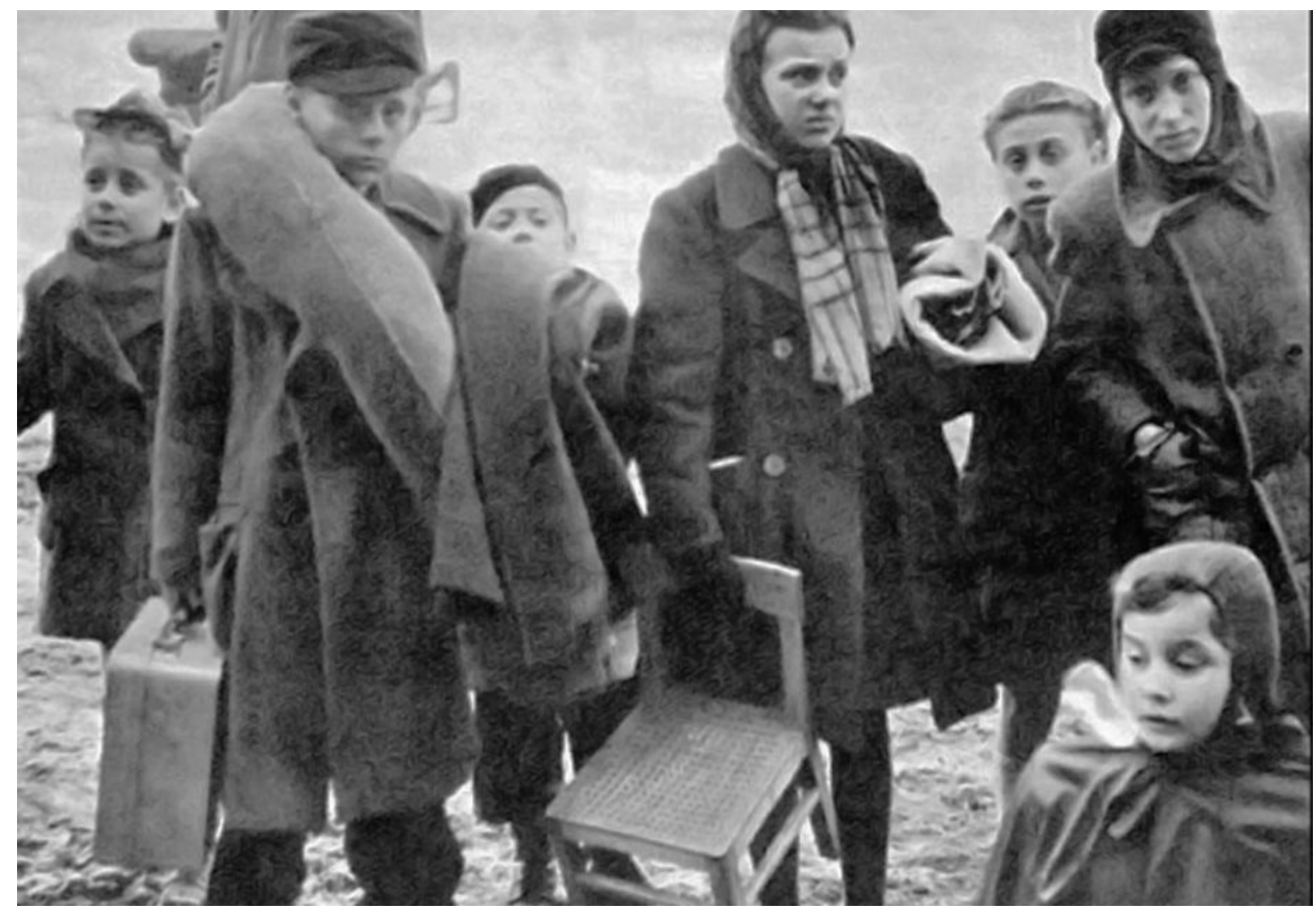

Esuli istriani

Foto tratta dal sito ISGREC

\begin{abstract}
Il progetto biennale Per la storia di un confine difficile: l'alto Adriatico nel Novecento, che ha coinvolto alcune scuole superiori della Toscana, rappresenta il traguardo - o la tappa intermedia di un lavoro sulla didattica del confine avviato dagli Istituti della rete toscana subito dopo l'istituzione del Giorno del ricordo. Il progetto ha offerto l'occasione di pensare didatticamente i molti temi sottesi alla questione del confine, fornendo nuove risorse per insegnare tanta parte del Novecento. Inoltre ha dato l'opportunità di ripercorrere il dibattito che accompagnò l'inserimento nel calendario civile del 10 febbraio e di riflettere insieme agli insegnanti sull'uso pubblico di quello che era - e non ha cessato di essere - un «passato che non passa».

L'esperienza didattica presentata intende offrire spunti per una riflessione critica sul confine orientale italiano, specie a fronte delle crescenti domande sul valore e i limiti di politiche e pratiche della memoria finora, però, concentrate prevalentemente sulla Shoah.
\end{abstract}




\section{Indice}

1. Il significato della complessità nella storia del confine orientale

\subsection{Spazio e tempo}

\subsection{Il confine non è una semplice linea}

\subsection{Voci dal confine}

2. La prospettiva del tempo presente

2.1. I tempi della memoria

\subsection{Il ritorno del territorio}

3. Uso pubblico della storia e public history

3.1 Paradigmi e memoria pubblica

3.2 Oltre possibili mistificazioni, verso una "public history di confine"

4. Per una pedagogia della memoria, tra formazione ed esperienza didattica

4.1 Sapere e saper fare: percorsi di formazione dei docenti toscani

\subsection{Conoscenze-competenze per i docenti}

\subsection{La funzione del viaggio nella pedagogia della memoria}

4.4. Il protagonismo degli studenti

4.5. È tempo per un bilancio?

La mostra virtuale a conclusione del progetto biennale Per la storia di un confine difficile: l'alto Adriatico nel Novecento della rete degli Istituti toscani con il coordinamento dell'ISGREC, è visibile al link: https://viaggiosulconfine.weebly.com

Il significato della complessità nella storia del confine orientale

\section{Spazio e tempo}

È una storia "complessa", si dice, di una speciale complessità rispetto a quella che senza alcun dubbio ogni storia nasconde.

Lo spazio è quello di confine, un confine particolare ma incomprensibile senza una comparazione con gli spazi dei confini europei. Già nel 2005, nel primo anno di celebrazione del Giorno del ricordo, fu provvidenziale un piccolo libro: Il dolore e l'esilio[1], pensato da Guido Crainz per la formazione degli insegnanti. Contiene la sintetica rappresentazione del fenomeno dell'esodo, che seguì ambedue le guerre mondiali, ma nel secondo dopoguerra determinò lo spostamento di milioni 
di cittadini europei, abitanti in zone di confine. In uno spazio relativamente piccolo, nel breve tempo della Seconda guerra mondiale, troviamo le tracce di una grande densità di avvenimenti, categorie diverse di fenomeni. Un esempio: la triplice forma del sistema concentrazionario: i campi per slavi dell'Italia fascista, come Gonars; la Risiera di San Sabba, luogo di transito per ebrei, di morte per oppositori politici e slavi; i campi di Tito, per oppositori politici e comunisti dissidenti.

Il tempo non si esaurisce nei decenni della guerra e del pur lungo dopoguerra. Violenze estreme, odi e stravolgimento del tessuto sociale di zone rurali e città rinviano a fenomeni di lunga durata, che precedono non solo il fascismo e la Grande guerra, l'irredentismo e l'Ottocento delle nazioni, ma anche Trieste porto asburgico. Nelle città istriane, oggi croate, nel Novecento si è rovesciato il rapporto fra le etnie: gli italiani sono minoranze, anche esigue, come rivela l'immagine di interi quartieri abbandonati in città come Albona. Le campagne istriane conservano segni d'abbandono, mentre nelle zone costiere colpisce il contrasto fra le località trasformate dal turismo e a breve distanza le residue memorie dell'antica presenza veneziana. Un filo fra i tanti da seguire nel lungo periodo è la lingua. A Trieste, massima espressione di una convivenza multietnica, mutata ma non scomparsa, in passato diverse lingue erano state patrimonio di gruppi, talvolta corrispondenti a funzioni sociali, in un'integrazione diventata via via sempre meno facile; oggi una minoranza significativa è quella slovena. L'Istria conserva lingue e dialetti sottostanti la attuale distinzione fra tre Stati nazionali - Italia, Slovenia e Croazia -: l'istro-veneto e l'istrioto nel sud.

\section{Il confine non è una semplice linea}

Il confine è di per sé un oggetto complesso; non è "una semplice linea", ma mette a confronto geografia e storia:

luogo di elezione delle guerre fra Stati, destinato a conservare anche dopo la conclusione dei conflitti ferite difficili da curare, in ragione dell'esistenza di frontiere culturali e mentali che sembrano più durevoli di quelle fra Stati[2].

Per chi vive su un confine il problema dell'identità ha una forma particolare. Le due sponde sono tanto nette per il cartografo quanto difficili da segnare, soprattutto per quello che è stato definito "confine mobile", tra Italia e gli attuali Stati, eredi di un secolo di drammatiche metamorfosi di tutta l'area balcanica. Lo hanno via via ridefinito i trattati, frutto di lavorii delle diplomazie, lunghi trent'anni, dopo la Seconda guerra mondiale.

Al linguaggio dei documenti e dei luoghi, così difficile da decifrare per i segni di memorie necessariamente divise, si aggiunge il racconto del vissuto di donne e uomini, una fra le fonti di questa storia.

\section{Voci dal confine}

Un istriano di Pola ci ha stupiti parlando di api e non di uomini, per spiegare da naturalista la sua visione del confine e della "identità":

...dalle bocche del Timavo per andare oltre c'è una barriera geo-biologica. Oltre il Timavo le api sono diverse. Qui ci sono le api istriane, descritte già nell'Ottocento da Monsignor Moscardin, che le ha chiamate istro-dalmatiche. L'ape istro-dalmatica ha un bel corsetto giallo, sa volare con la bora e col neverin.

Le api istriane sono una specie da conservare, come la cultura di chi ha abitato per secoli l'Istria. Il testimone Livio Dorigo si definisce italiano e italiana è l'unica lingua che dichiara di parlare; 
ricorda le radici risorgimentali dell'italianità della sua famiglia e con durezza condanna $i$ nazionalism $i$, responsabili della tragedia delle terre di confine. Profugo con la famiglia dal 1947, è tornato nelle sue zone ed è impegnato nella promozione di una cultura dei luoghi e della memoria, per trasmettere una visione cosmopolita. Attraverso le api propone una sua filosofia: la conoscenza della natura e della storia come antidoto a divisioni e rancori, perché «la conoscenza scaccia la paura».

Un'altra voce è quella di Claudio Magris, triestino, autore di pagine tra le più belle di letteratura e memorialistica di confine. Ne L'infinito viaggiare, fissa le due verità del confine: il bisogno di «Heimat, la casa natale, la patria», sentimento comune a chi ha scelto l'esodo e a chi è rimasto, ma insieme il rifiuto del confine come frontiera invalicabile:

Alle genti di una riva quelle della riva opposta sembrano spesso barbare, pericolose e piene di pregiudizi nei confronti di chi vive sull'altra sponda. Ma se ci si mette a girare su e giù per il ponte, mescolandosi alle persone che vi transitano e andando da una riva all'altra fino a non sapere più bene da quale parte e in quale paese si sia, si ritrova la benevolenza per se stessi e il piacere del mondo. "Dov'è la frontiera" chiede Saramago sul confine tra Spagna e Portogallo ai pesci che, nello stesso fiume, nuotano, a seconda che guizzino vicino a una sponda o a un'altra, ora nel Duero ora nel Douro[3].

\section{La prospettiva del tempo presente}

\section{I tempi della memoria}

Nel dibattito politico non sono mai cessate né la denuncia dei lunghi silenzi né le accuse incrociate di intenzionale oblio di foibe ed esodo. Del resto, su questo piano, poco efficaci sono parsi sia il riconoscimento di monumento nazionale per il luogo-simbolo degli infoibamenti (Basovizza nel 1992) sia la legge istitutiva del Giorno del ricordo, nel 2004. Se nel tempo la memoria collettiva si è rimodellata e la storiografia ha fatto grandi passi, non tutti i nodi sono stati sciolti e i conti con il passato chiusi. Qui pesa anche la troppo lunga gestazione del Trattato di Osimo, nel 1975, che non ha sanato rancori. Il 10 febbraio, data scelta dalla legge istitutiva del Giorno del ricordo, continua annualmente a riportare alla luce le aspettative deluse dall'evento che evoca, il Trattato di pace del 1947.

Ma non tutti gli anni sono stati uguali. Gli itinerari della dialettica memoria-oblio nei decenni seguono le tappe della storia politica, i passaggi delle diplomazie e le cesure del secondo Novecento. Basta pensare alla caduta del muro di Berlino, alla "esplosione delle nazioni" nella Jugoslavia e all'ingresso nell'Europa dei nuovi Stati balcanici. Nel 1993, in un rinnovato quadro internazionale, si costituì la Commissione storico-culturale italo-slovena, che concluse i lavori nel 2000; rappresentò un passo significativo del cammino verso la normalizzazione delle relazioni fra $i$ due Paesi ma - come osserva la storica Marta Verginella - si trattò di una narrazione ancora nazionale, assai lontana dall'espressione ideale per un'area multietnica e di confine.

Si invera l'idea del presente che cambia il passato, fino alla recentissima, drammatica riemersione del tema delle frontiere, con il ritorno di nazionalismi e chiusure verso l'altro, l'estraneo che diventa il nemico, ora l'immigrato che bussa alle porte dell'Europa. In tutto il continente si sono manifestati «processi di costruzione delle narrazioni populiste, neonazionaliste, identitarie e sovraniste», essendo rimasti irrisolti «processi di "patriottismo della Costituzione", con il bipolarismo prima e poi con l'esaurimento di questo così come dell'ordinamento partitico postbellico»[4].

\section{Il ritorno del territorio}


Conviene qui tornare alla persistenza di memorie rancorose, anche nelle seconde generazioni di esuli. Non c'è che la storia per venirne a capo, tenendole come preziose fonti per non mollare con la fatica della continua reinterpretazione. Sarebbe una inutile semplificazione opporre come unici argomenti i principi umanitari e gli appelli moralistici. Nelle parole "giuste" di Livio Dorigo, malgrado il peso dello sradicamento dalla sua Pola, c'è l'invito alla costruzione di ponti in luogo di barriere, ma è forte anche il sentimento di appartenenza alla natura e alla cultura istriane. Nelle memorie di chi scelse di rimanere, di quanti abbandonarono "senza più tornare", dei pochi che hanno trovato la forza per un rientro, un filo lega atteggiamenti anche opposti verso il passato: il sentimento forte del territorio.

A vent'anni dall'inizio del Millennio, nel bene e nel male la globalizzazione è un processo irreversibile. Le reti hanno sostituito le linee; è più complicato l'equilibrio fra diversi centri di potere; ma gli individui si sentono più isolati e, sostiene in un libro recente Charles Maier, «...l'idea di territorio - che sembrava essere caduta in garbato disuso - si è riaffacciata con prepotente e rinnovata urgenza nelle nostre vite»[5]. Il rafforzamento dei legami fra comunità e territorio richiede ascolto e comprensione. In Europa si sono moltiplicate le spinte separatiste, talvolta indipendenti dalle parti politiche, è avvenuto il recupero di ideologie razziste e si discute di nuovi muri e filo spinato, mentre il Mediterraneo è conteso fra divieti e soccorso umanitario a migranti. Tuttavia, seguendo il filo dello studio di Charles Maier, « le frontiere sono più che delle semplici barriere; per alcuni, sono garanzia di comunità e di appartenenza».

La storia del confine alto Adriatico rappresenta dunque un caso di studio interdisciplinare affrontato dalla prospettiva del presente, che può essere di grande utilità sul terreno della didattica. Una didattica per l'educazione storica e una didattica per l'educazione civica.

\section{Uso pubblico della storia e public history}

\section{Paradigmi e memoria pubblica}

Ha quasi un decennio un'opera importante di Giovanni De Luna, che ruota intorno al «paradigma vittimario» delle «memorie di un'Italia divisa». Vi analizza «i meccanismi di costruzione della memoria pubblica nel nostro paese e ne documenta la contraddittoria fragilità»[6]. In un contesto europeo, le specificità dell'Italia sono ricondotte alla persistenza di nodi irrisolti della nostra storia e memoria e all'inefficacia della costruzione di un calendario della memoria fitto di date che non riescono a creare le basi per rifondare una cittadinanza. Debolezza della politica, debolezza dello Stato, scriveva De Luna in un libro dedicato a Nicola Gallerano, che fra gli anni Ottanta e Novanta ci aveva insegnato a distinguere tra improprie strumentalizzazioni di parte e un «uso pubblico della storia consapevole e critico, capace di mettere in questione l'opacità e l'eternità del passato per riscattarlo dalla tirannia del presente»[7]. Malgrado la fiducia nella disciplina che essi praticano, non la giudicano condizione sufficiente per istituire un utile rapporto con il passato.

Le occasioni di un impegno intenso sulla questione iniziarono in Toscana dopo la legge del 2004, ma ne aveva parlato già Raoul Pupo in un convengo a Grosseto, nel 1996. La rete degli Istituti toscani ha avviato fin dal 2005 una serie di esperienze con insegnanti e scuole toscane, fino all'ultimo progetto biennale, sperimentato nel 2017-18, poi ripetuto nel biennio successivo e auspicabilmente a regime, su proposta del Consiglio regionale e inserito dalla Giunta nel quadro delle politiche sulla memoria regolate dalla 1. r. 38/2002.

\section{Oltre possibili mistificazioni, verso una "public history di confine".}


Questo non ha impedito l'emergere nello stesso Consiglio regionale di polemiche politiche, fino allo scorso febbraio, quando un deputato, proprio l'ex-consigliere presentatore della mozione che nel 2016 aveva portato al progetto, minacciò un esposto alla procura contro la Regione per "negazionismo". Non c'erano le condizioni perché ci fosse un seguito; in linea con una sequenza ormai consueta, si è passati dalla furia mediatica nei dintorni del 10 febbraio al silenzio. Consultare la rete dimostra comunque quanto stretto sia il sentiero: si sono sommate critiche di alcuni membri di associazioni di ex-profughi, ignari della natura del lavoro in corso, ed errate interpretazioni, diffuse dal collettivo che si firma con lo pseudonimo Nicoletta Bourbaki. A Grosseto, da qualche anno si ripete l'assurda assimilazione alle foibe di un episodio locale, che l'ISGREC è accusato di occultare: fascisti gettati durante la guerra in una cavità, nel lessico familiare del paese (Roccastrada), pozzo sprofondatoio.

Esodo e foibe hanno risentito del clima incandescente della retorica dell'italianità, delle frontiere da chiudere e del montare di un antieuropeismo. Si alimenta un sentimento (la paura), che evoca per contrasto la filosofia del profugo Livio Dorigo: «La conoscenza scaccia la paura». Potere della conoscenza, se le interpretazioni sono documentate e sottoposte al vaglio della critica della fonte, impotenza dell'uso rigoroso dei paradigmi della disciplina storia, in presenza di un'invasione nei media di falsificazioni del passato credibili, confezionate utilizzando esempi storici più o meno casuali. La contiguità delle date (Memoria 27 gennaio e Ricordo 10 febbraio) ha scatenato la competizione fra le memorie: alcune amministrazioni toscane hanno subordinato la cittadinanza onoraria alla ex-deportata Liliana Segre a quella all'esule giuliana Egea Haffner.

Quale ruolo ha giocato in questo contesto l'uso della public history? Come si dirà in seguito, una produzione culturale rivolta al pubblico scolastico e alla cittadinanza attraverso l'uso di diversi mezzi e linguaggi ha aggiunto strumenti nuovi a quelli tipici della comunicazione di ricerche scientifiche e alla produzione didattica interna al lavoro nelle scuole. Il viaggio è stato un segmento importante della produzione di una "public history di confine". La congiuntura della pandemia in atto ci spinge a impiegare al meglio gli strumenti che abbiamo già da mettere in campo e a cercarne di nuovi, con il massimo sforzo per evitare rischiose improvvisazioni.

\section{Per una pedagogia della memoria, tra formazione ed esperienza didattica}

\section{Sapere e saper fare: percorsi di formazione dei docenti toscani}

Un dato interessante è la risposta positiva degli insegnanti fin dal 2005 alle proposte degli Istituti toscani, sostenute dalla Regione Toscana e dall'USR toscano. In un quindicennio si sono consolidate e diversificate iniziative che sembrano aver inciso realmente nella programmazione didattica[8].

Nel limitato perimetro della Toscana, abbiamo avuto a disposizione frammenti importanti di storia e memoria. C'era stata la presenza silenziosa di profughi in diversi campi di accoglienza - a Firenze nel corso degli anni ne furono accolti 1.692 nel Campo Profughi della ex Manifattura Tabacchi. Figura importante è stato Sergio Rusich, che trasformò l'esilio in un impegno civile e culturale. Firenze ha avuto un legame culturale forte con Trieste. Negli anni Ottanta il Gabinetto Viesseux dedicò a questo tema una mostra, Intellettuali triestini a Firenze (1900-1950). Umberto Saba ricorda gli anni difficili dell'esilio a Firenze dalla sua Trieste, per sfuggire alle persecuzioni nazifasciste («M’hai celato nei dì della sventura»).

In sintesi presentiamo alcuni caratteri del lavoro toscano: 
1. progettualità di lungo periodo: corsi, seminari e iniziative di altro genere sono stati pensati in una sequenza pluriennale;

2. progressiva costruzione di un sistema a rete, realizzata decentrando parzialmente la formazione ma coinvolgendo direttamente le scuole;

3. modello adottato: formazione di insegnanti formatori;

4. produzione di strumenti permanenti: un primo volume di materiali per la didattica, un documentario, una mostra itinerante e sollecitazioni per ricerche storiche locali, come lo studio di Laura Benedettelli sull'inserimento di profughi a Grosseto;

5. largo spazio a iniziative di formazione valorizzate sia dalla presenza di storici di alto profilo scientifico, per colmare i vuoti di conoscenza, sia da riflessioni mirate su confine e memoria pubblica;

6. frequente ricorso a laboratori storici, utili alla costruzione di competenze didattiche, e centralità del tema della pluralità delle fonti;

7. sperimentazione di due viaggi di studio rivolti ad un piccolo numero di insegnanti nell'area del confine e con meta a Sarajevo, che hanno preceduto l'esperienza del viaggio degli studenti.

8. La Summer school per il piccolo gruppo di insegnanti selezionati per il viaggio del secondo progetto ha cercato di tenere insieme il fine della conoscenza storica, la ricerca didattica e il tema della comunicazione. Si sono alternate lezioni di storici, laboratori gestiti da insegnanti divenuti formatori, programmazione di attività con gli studenti con la guida di esperti di media e la collaborazione di associazioni di ex-profughi.

\section{Conoscenze-competenze per i docenti}

Molta letteratura sulla trasmissione di storie del Novecento, legate alla memoria pubblica e alle politiche della memoria, è incentrata su Auschwitz, declinato prevalentemente come storia-memoria della Shoàh. Ma Auschwitz stesso può essere un efficace termine di riferimento per una comparazione con le complesse vicende del confine nord-orientale italiano; e questo soprattutto in ragione della maggiore maturità critica raggiunta rispetto alla riflessione sull'alto Adriatico che malgrado la legge istitutiva del Giorno del ricordo segua di soli cinque anni quella della Memoria appare ancora debole e irta di fragilità.

Anche su questa rivista è stata messa in discussione l'ipertrofia della memoria, con il corollario della falsa equazione: ricordare = vaccinarsi dall'orrore[9]. La trasmissione della storia della Shoàh è stata spesso schiacciata sul testimone, massima espressione del trasferimento in didattica del «paradigma vittimario». Le liturgie commemorative hanno finito per trasferire anche nella scuola le loro retoriche. Fino all'ultima domanda «che fare, ora che è finita l'era del testimone?»

Nelle storie di confine, la pluralità dei temi e delle fonti rende meno invadente il testimone. Nel rapporto storia-memoria, la bilancia qui pende dalla parte della storia e ha uno spazio ineludibile il contesto europeo; al monopolio della soggettività della voce narrante si contrappone il confronto memorie individuali-collettive. Vale la pena citare, come tappa del nostro cammino, il progetto europeo Memorie europee di frontiere, il cui partner italiano è stato l'ISGREC[10]. Sono state messe a confronto le storie di diversi confini, ci si è dovuti misurare con le dinamiche delle violenze e i tempi diversi dell'esilio, per concludere con le guerre balcaniche degli anni Novanta. Pur rimanendo il confine (orientale per noi) il prezioso «laboratorio per la storia del Novecento», abbiamo a che fare con la pluralità e maggiore complessità di fonti, storie nazionali e implicazioni con il presente dell'Europa, il che rende più facile assumere il punto di vista dell'altro. I concetti di assoluto - il male - o di unicità - la Shoàh - lasciano spazio alla relativizzazione, che riporta alla misura della storicità. 
Trasmettere tanta complessità richiede padronanza di metodo storico e uso competente delle fonti. Non è banale la competenza sui linguaggi dei media, se c'è da decodificare le ragioni di un dibattito pubblico che, soprattutto con gli studenti più grandi, può raggiungere le classi. Nell'intervallo fra la Summer school dell'agosto 2019 e il viaggio, venne distribuito nelle sale cinematografiche e in televisione Rosso Istria, film-biografia di Norma Cossetto, costruito come "rivelazione" dell'orrore delle foibe attraverso sequenze d'intollerabile violenza. Nella discussione pubblica intervennero le associazioni di esuli giuliano-istriano-dalmati, diverse tra loro per approccio culturale, ma nella cui scala di valori prevale il dolore per l'oblio, che ha coperto a lungo sofferenze e privazioni. Il gruppo di insegnanti intervenuti ebbe l'occasione di entrare in relazione con la dimensione politica, per una comparazione con il prodotto di un'altra epoca: Il cuore nel pozzo, fiction RAI trasmessa nel febbraio 2005 e seguita da una bagarre politico-mediatica. Due prodotti mediatici diversi per impianto e qualità ma accomunati dall'identico il problema: la correlazione fra sapere storico e una pluralità di altre competenze necessarie alla sua trasmissione. Con, in più, la necessità di fare i conti con la rilevanza politica del tema dei confini, cresciuta con rapidità imprevedibile e oggetto di una conflittualità politica ad alta intensità. Il lavoro in classe richiede all'insegnante anche una gestione della personale coscienza di cittadino/a, la capacità di prendere posizione e la responsabilità di fare scelte. Gli insegnanti hanno dovuto decidere se accettare o rifiutare proposte di proiezione di Rosso Istria organizzate da Enti locali e molti si sono chiesti comunque se utilizzarlo come fonte.

\section{La funzione del viaggio nella pedagogia della memoria.}

Per ogni viaggio serve un vademecum che gli dia senso, prima delle guide esperte, in situazione.

Non c'è viaggio senza che si attraversino frontiere - politiche, linguistiche, sociali, culturali, psicologiche, anche invisibili... Viaggiare non vuol dire soltanto andare dall'altra parte della frontiera. Ma anche scoprire di essere sempre pure dall'altra parte[11].

Abbiamo scelto queste parole di Claudio Magris per accompagnare i ragazzi a porsi e porre a noi domande, che ci sembra possano racchiudere una parte del significato dell'esperienza da fare ancor prima della partenza.

Viste nel loro insieme, ciascuna nella propria specificità, le stazioni del percorso sono espressione della storicità della memoria. Da imponenti monumentalizzazioni a tracce tardive, rimedio di un lungo oblio, a memorie plurime dello stesso evento nel medesimo luogo, a segni di completo e irreparabile abbandono.

Nessun luogo ha l'unicità di Auschwitz, nessuno ha assunto un valore di simbolo comparabile. Non lo è nemmeno Basovizza, nonostante ospiti le commemorazioni ufficiali ogni 10 febbraio e rappresenti la memoria del più citato fra i ricordi incastonati nelle città o nei paesaggi di confine: le foibe. Malgrado non sia una vera foiba, lo spazio sovrastante il pozzo dove sono stati gettati e ritrovati corpi è stato scelto come monumento d'interesse nazionale

Diversità di luoghi qui significa linguaggi diversi. Arte e memoria per Basovizza e Redipuglia, prima tappa per cronologia (i caduti della Grande Guerra): ambedue con la forma che ha voluto dargli lo "spirito del tempo" della costruzione. Nel sacrario di Redipuglia il lutto per i caduti è stato trasformato nell'esaltazione della retorica nazionale dell'eroismo in guerra.

La vera foiba Plutone - l'imboccatura di una piccola voragine -, Magazzino 18 - cumuli di masserizie lasciate dagli italiani che abbandonarono tutto, anche gli oggetti della vita quotidiana -, un intero quartiere ad Albona di case chiuse da lucchetti arrugginiti, rimasti intatti dagli anni Quaranta sono luoghi «conservati come puri fatti che parlano da soli». In un saggio datato, ma di 
una straordinaria efficacia, Sarah Farmer parla di «resti materiali e memoria». Le rovine del villaggio di Oradour sur Glane, incendiato da un reparto di SS dopo il massacro di 642 abitanti, sono «paesaggio educativo». La materialità dei resti fisici è stata lasciata a raccontare «una storia che insegna qualcosa a chi si ferma a guardarli» [12].

La memoria si conserva attraverso luoghi trasformati o si lascia che parlino da soli e creino così «un paesaggio educativo»? In questo caso si cristallizza la memoria con l'emozione. Nell'esperienza del viaggio sul confine si è sperimentata l'interazione fra la memoria di pietra dei monumenti o il paesaggio che evoca l'evento, la memoria pubblica (le istituzioni e gli storici) e la voce di chi ha vissuto o ha raccolto il racconto di chi ha vissuto, per consegnare la memoria alla storia. Voci spesso dissonanti negli stessi luoghi hanno educato gli studenti a misurarsi con la ricchezza e insieme con il difficile uso delle fonti di memoria.

\section{Il protagonismo degli studenti}

Finalmente in carne e ossa nei giorni del viaggio, in realtà gli studenti sono stati sempre presenti, sia nelle fasi preliminari sia al termine dell'esperienza didattica: dalla narrazione fatta dagli insegnanti di quanto appreso durante la Summer, alle prove di lavoro svolte in classe, fino al ritorno, per una effettiva disseminazione del sapere storico e delle competenze acquisite.

Nei cinque giorni sul confine, gli studenti che hanno partecipato al viaggio sono stati due per insegnante, ma tanto ciascuna delle loro classi quanto, in buona parte, le rispettive scuole hanno ampiamente fruito di quel che è stato creato attraverso il progetto.

Se in altre precedenti esperienze la cura della produzione permanente di strumenti per la didattica era stata affidata al gruppo di esperti e insegnanti, il viaggio del febbraio 2020 ha ribaltato l'approccio tradizionale. Cuore pulsante del progetto sono stati gli studenti che hanno elaborato il «giornale di bordo»: testi, immagini, note critiche, preziosi materiali che rimangono. Due giornalisti e un fotografo-videomaker hanno documentato il percorso formativo degli insegnanti e li hanno accompagnati durante il viaggio.

La singolarità è consistita nel sistematico svolgimento di un ruolo attivo, coerente con le vocazioni personali di ciascuno, in ogni tappa del viaggio. Il piccolo numero è stata una variabile fondamentale; non era scontato passare dall'idea alla concretezza: dare spazio alle abilità dei ragazzi nella manipolazione del digitale e della rete, uscire con materiali interamente prodotti da loro, in modo tale che assumessero, accanto agli insegnanti, il ruolo di mediatori nella trasmissione di conoscenze e competenze alla classe e alla scuola. Non era nemmeno scontato che manifestassero un autentico interesse e fossero in grado di superare l'iniziale disorientamento, normale per i viaggiatori del confine. Invece, in presenza, hanno saputo reagire all'evidenza di conflitti di memorie. Nella scrittura dei testi e nelle interviste hanno per lo più colto la diversa natura della storia rispetto alla memoria.

Una spia per giudicare se e quanto l'esperimento abbia funzionato è stata la curiosità, che si è tradotta in frequenti domande e in una costante interazione con gli esperti, documentata anche dalle loro rielaborazioni.

In particolare, il momento in cui gli studenti sono diventati protagonisti consapevoli è stato l'incontro con la scuola italiana di Fiume, penultima tappa in Istria. Più che l'affermazione della propria identità italiana richiesta dal contesto istituzionale - Console italiano a Fiume, Associazione degli italiani e Preside della scuola - è emersa una sorta di complicità, che si è manifestata nella coscienza di appartenere a mondi lontani ma dialoganti. Fiume è stata per tutti un momento di 
confortante sintonia con italiani che, pur lamentando di essere minoranza in una città dal passato italiano (valga ad esempio lo scarso coinvolgimento nei programmi di Fiume capitale europea della cultura), sono immersi in un clima sociale positivo e godono di spazi comunitari adeguati. Una buona lezione è stata inoltre fornita dall'osservare una integrazione, non perfetta ma positiva, dei ragazzi italiani con i coetanei croati. Le due frontiere che si attraversano per passare dalla regione di Trieste a Fiume (quella croata è una vera e propria frontiera) sono un dato di fatto ed entrano a pieno titolo tra gli argomenti che caratterizzano le questioni degli immigrati, del Mediterraneo e delle altre rotte verso il nord Europa che risalgono i Balcani.

Un'esperienza non è un laboratorio sufficientemente attrezzato per verificare la ripetibilità del progetto ma, fatte salve alcune correzioni, sembra che il modello possa funzionare.

\section{È tempo per un bilancio?}

È impossibile prevedere il futuro di progetti e attività della scuola, fin quando non sarà chiara l'evoluzione della congiuntura sanitaria attuale. Possiamo tentare una ricognizione su quel che resta in termini di strumenti permanenti, ma rinviare a un tempo successivo la riflessione sulla didattica in classe, che potrebbe consegnarci solo chi ha sedimentato esperienze sul campo.

In queste pagine si è cercato di mostrare genesi e tappe di un percorso, di cui l'ultimo progetto è il punto di arrivo. Una scelta fondamentale, divenuta nel tempo sempre più consapevole, è stata la produzione di strumenti di public history, partendo dalla relazione fra ricerca e didattica. Se ne è estesa la diffusione non solo con il documentario storico frutto del progetto biennale 2017-18, utilizzato in classe, ma anche con un'ampia distribuzione pubblica.

Lo spazio delle iniziative istituzionali era stato giudicato importante, anche alla luce di un giudizio quasi unanime di insufficienza della ripetizione di liturgie celebrative nel Giorno del ricordo. È piuttosto lunga la lista di Comuni o Prefetture con cui si è instaurato un rapporto di collaborazione, che in passato era prevalentemente limitato ad altre date del calendario civile.

L'ultima esperienza ha lasciato un nuovo patrimonio; è un valore aggiunto, per essere stato una creazione non estemporanea dei cinquanta studenti, diversa da quel che avviene ordinariamente per qualsiasi esperienza di visita. Sul sito di Radiocora è on line il «giornale di bordo» e la Domus mazziniana di Pisa ha in rete i lavori con cui gli studenti pisani hanno rielaborato autonomamente quanto hanno tratto dall'esperienza. Riflessioni critiche, immagini, interviste, riprese video: l'ampio dossier, elaborato dalle ragazze e dai ragazzi che hanno partecipato al viaggio, è consultabile e scaricabile alla pagina «Il diario degli studenti» del sito Storia di un confine difficile[13]. Si tratta di materiali didattici che confermano l'efficacia e la validità di progetti finalizzati ad integrare il sapere storico e le conoscenze sui luoghi della memoria con le competenze necessarie alla loro trasmissione e comunicazione.

Un bilancio è da fare. I dati e le parziali considerazioni suscitate da quest'esperienza possono offrire le condizioni per un discorso critico su quanto (e come) si possa trarre da storia e memoria del confine alto Adriatico? È tempo di farlo? 
[1] G. Crainz, Il dolore e l'esilio. L'Istria e le memorie divise d'Europa, Donzelli, Roma 2005.

[2] L. Benedettelli, M. Fiorani e L. Rocchi, Per una storia del confine orientale, ISGREC, Grosseto 2007.

[3] C. Magris, L'infinito viaggiare, Mondadori, Milano 2005, pp. XI-XIII.

[4] C. Vercelli, Ad oriente dell'Occidente. Il confine orientale tra storia e memorie nella costruzione dell'identità repubblicana, relazione nella Summer school di Grosseto, 26 agosto 2019.

[5] C. Maier, Dentro i confini. Territorio e potere dal 1500 a oggi, Einaudi, Torino 2016, p. 3.

[6] G. De Luna, La Repubblica del dolore. Le memorie di un'Italia divisa, Feltrinelli, Milano 2011, pp. 9-13

[7] N. Gallerano, Le verità della storia. Scritti sull'uso pubblico del passato, Manifestolibri, Roma 1999, p. 54.

[8] Un esempio è il programma dell'anno scolastico 2009-2010: ciclo di lezioni-laboratorio e seminari decentrati in ogni provincia, dove i dieci insegnanti coinvolti nella prima formazione e nel viaggio svolsero il ruolo di formatori.

[9] In queste pagine i temi della memoria e dei luoghi sono già stati affrontati con numerose riflessioni critiche (cfr., fra gli altri, gli articoli di Valentina Pisanty, Che cosa è andato storto? Le politiche della memoria nell'epoca del post-testimone, e di Alberto Cavaglion, Paesaggi contaminati da decontaminare. Qualche riflessione sui luoghi della memoria, in novecento.org, $\mathrm{n}$. 13, febbraio 2020).

[10] Mémoires européennes des frontières è un progetto europeo (misura "Remembrance 2016", Capofila l'European Observatory of memoires). Il convegno conclusivo si è svolto a Grosseto, nel febbraio 2017.

[11] C. Magris, ivi, p. XII.

[12] S. Farmer, Le rovine di Oradour sur Glane, in "Le parole e le cose", n. 9 ,1995, pp. 155-168.

[13] Cfr, https://viaggiosulconfine.weebly.com/il-diario-degli-studenti.html 